\title{
Low-cost hydrogen sensor in the ppm range with purely optical read-out
}

Ediz Herkert, Florian Sterl*, Nikolai Strohfeldt, Ramon Walter, and Harald Giessen

4th Physics Institute and Research Center SCoPE, University of Stuttgart, Pfaffenwaldring 57, 70569 Stuttgart, Germany

${ }^{*}$ Corresponding Author: f.sterl@pi4.uni-stuttgart.de

Keywords: hydrogen, hydrogen detection, plasmonics, optical sensing, large-area nanofabrication, palladium, thermodynamics. 
a

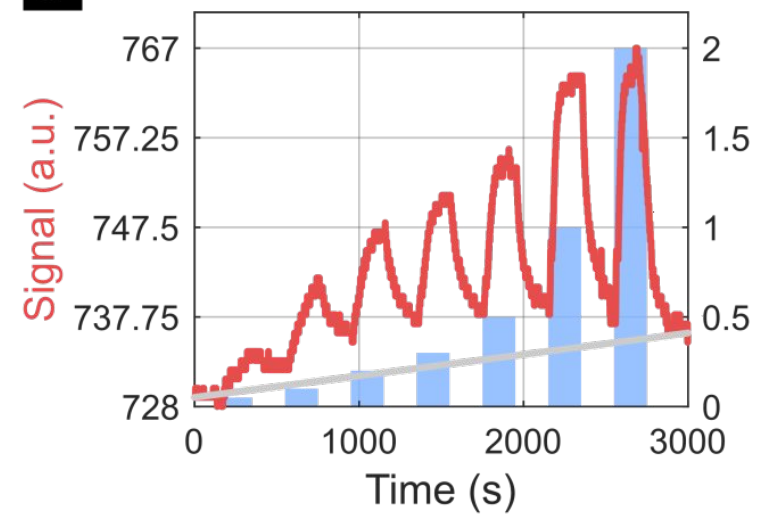

b

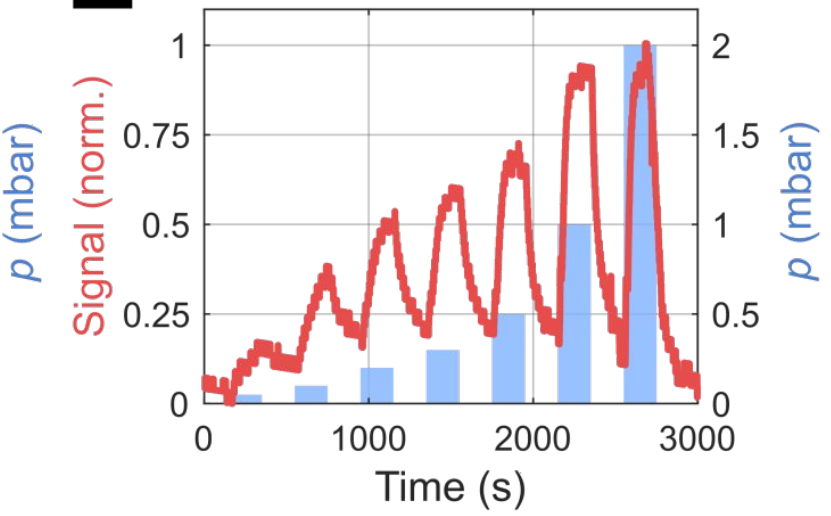

Figure S1. Linear baseline correction and normalization process. (a) The raw reflected intensity (arbitrary units) exhibits a baseline drift. The drift is corrected by the linear gray line that connects the first and the last measurement point. (b) The corrected signal is obtained after subtracting the linear baseline and normalizing the values between 0 and 1 .

The measured reflected intensities that are presented in this work are subsequently baseline corrected and normalized. A simple linear baseline correction is used so that the reader gets a clear impression of the nonlinear baseline shifts that might occur due to incomplete dehydrogenation of the palladium nanodisks. For the linear fit, only the first and the last datapoints in the displayed datasets are used. Afterwards, the linear curve is subtracted from all datapoints in the dataset. Finally, all values are normalized such that the minimum value is at 0 and the maximum value is at 1 . Fig. S1 demonstrates how the baseline correction and normalization is carried out for the raw dataset shown in Fig. 4 (a). 

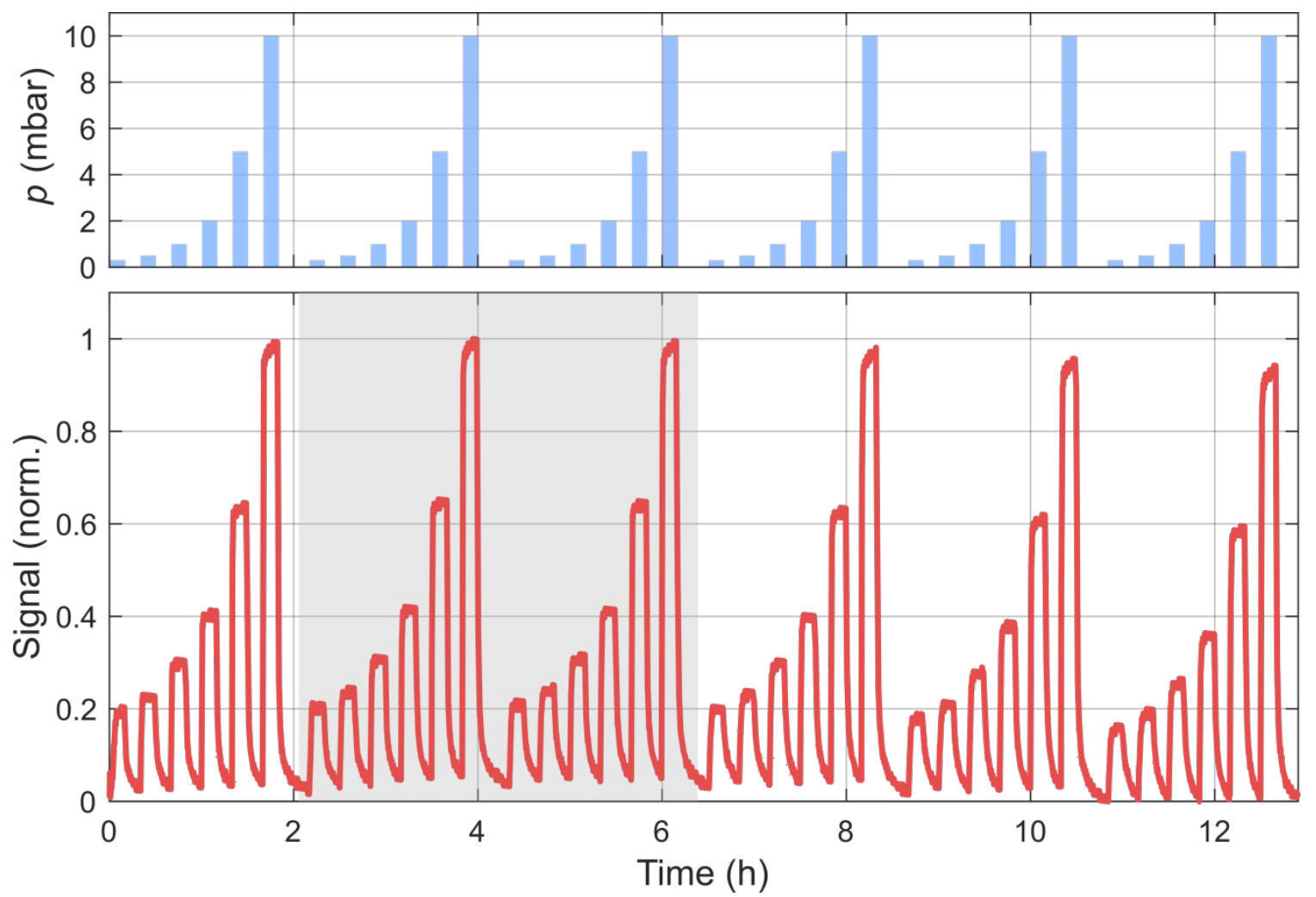

Figure S2 Short-term signal stability. The signal remains very stable over a time of more than 12 hours and a hydrogen concentration range from $300-10,000 \mathrm{ppm}$. The gas exposure time is $600 \mathrm{~s}$ so that full palladium dehydrogenation and thus signal recovery is warranted. The gray shaded area indicates the sequence shown in Fig. 4 (b). The signal is linearly baseline corrected and normalized.

Fig. S2 depicts the full measurement cycle from which the data in Fig. 4 (b) is extracted. This subset of data corresponds to the signal that is measured between $\sim 2 \mathrm{~h}$ and $\sim 6.4$ $\mathrm{h}$ (see gray shaded area in Fig. S2). As mentioned in the main body of this work, the signal stays very stable over more than $12 \mathrm{~h}$. This indicates excellent short-term signal stability at low hydrogen partial pressures ranging from 0.3 mbar to 10 mbar (300 $10,000 \mathrm{ppm})$. 
a
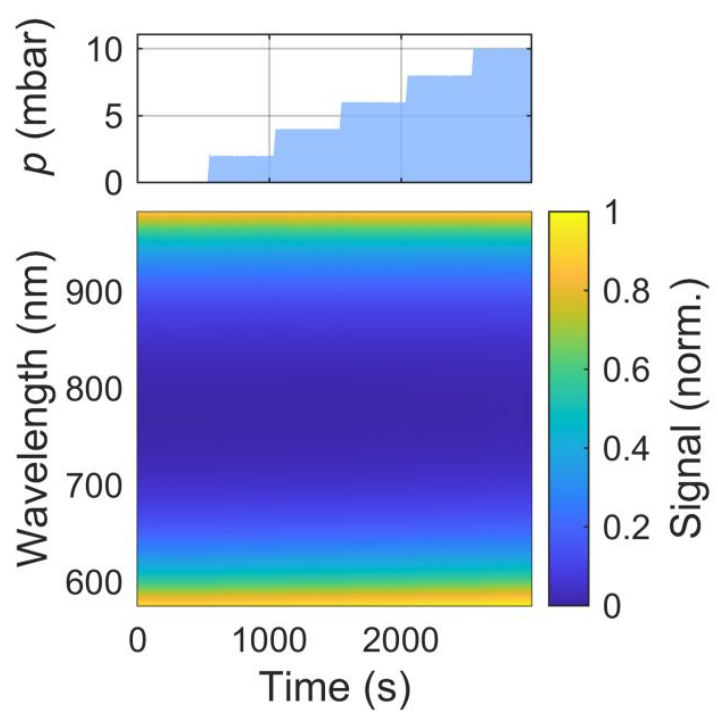

c

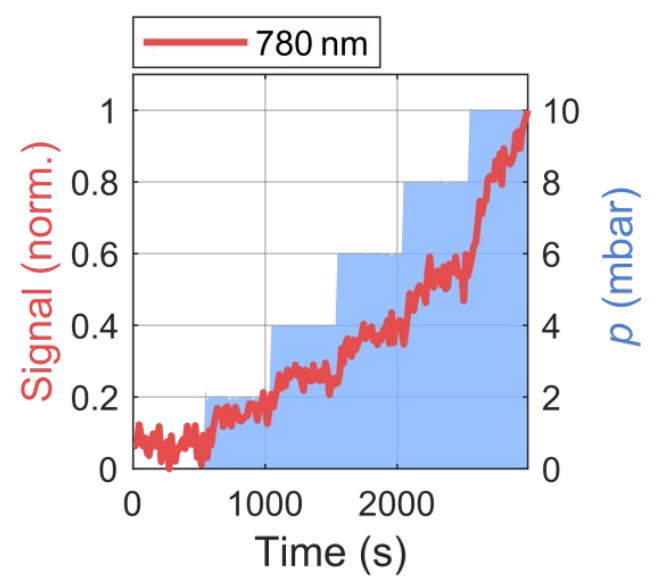

b
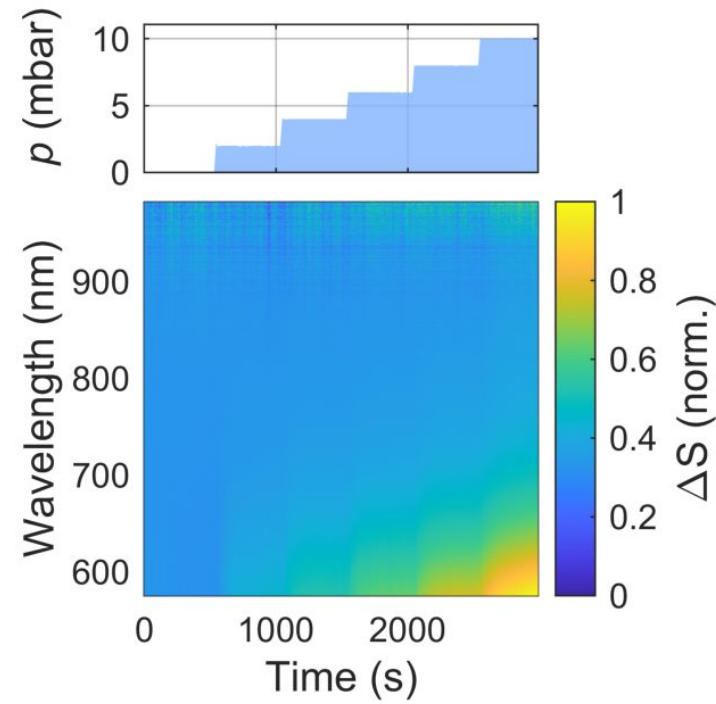

d

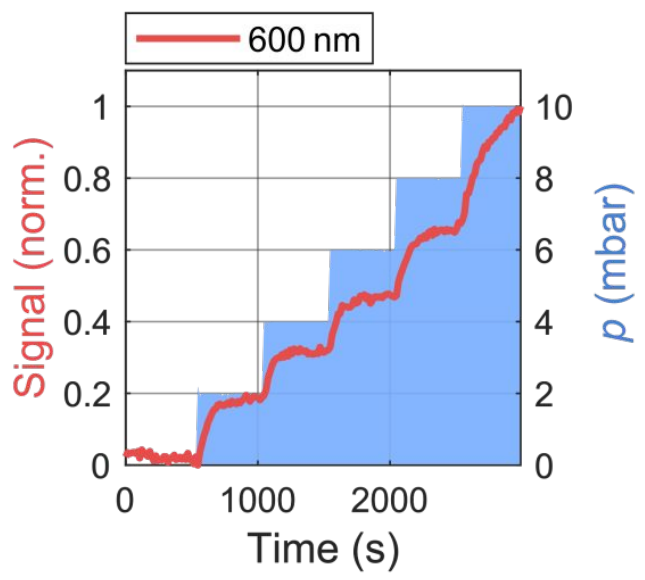

Figure S3. Influence of the read-out wavelength. The data shown here is recorded with a brightfield microspectroscopy setup that provides the spectral properties of the reactive sample at different hydrogen partial pressures. The recorded spectra at temporally varying hydrogen partial pressures are shown in (a). The minimum signal lays around $780 \mathrm{~nm}$. (b) By subtracting the first spectrum (at $p=0$ mbar) from each spectrum the signal change $\Delta S$ is determined. The strongest signal change is found to be around $600 \mathrm{~nm}$. (c) and (d) emphasize the importance of the read-out wavelength, since the signal-to-noise ratio and thus the sensitivity of the sensor is strongly enhanced at $600 \mathrm{~nm}$. Obviously, it must be accounted for the noise in the compact sensor unit to optimize the sensor sensitivity. 
The optimal read-out wavelength for the reflectance measurements can be determined from spectroscopic measurements. For this, the sample must be exposed to different hydrogen concentrations while the time and spectrally resolved reflected intensities are measured. This is accomplished with a customized microspectroscopy setup that records the spectrally resolved reflectance at a certain location on the sample. The absolute signal change $\Delta S(\lambda, p)=S(\lambda, p)-S(\lambda, p=0)$ can be determined by subtracting a reflectance spectrum at zero hydrogen partial pressure from all recorded spectra. Eventually, the optimal wavelength is determined by selecting the time series with maximal absolute signal change in the desired hydrogen concentration range. It is important to stress that the sensitivity of the sensor critically depends on the signalto-noise (SNR) ratio of the measured signal and not only the maximal signal change. Because of that, particularly strong noise within a certain spectral range can shift the optimal wavelength towards wavelengths with non-maximum signal changes. Nevertheless, the discussed wavelength selection procedure yields a significantly enhanced sensitivity in most of the cases. Fig. S3 (c) and (d) demonstrate the substantial signal improvement that is obtained if the read-out wavelength is chosen according to this selection procedure $(600 \mathrm{~nm})$ and not at the resonance wavelength (780 nm). 
a

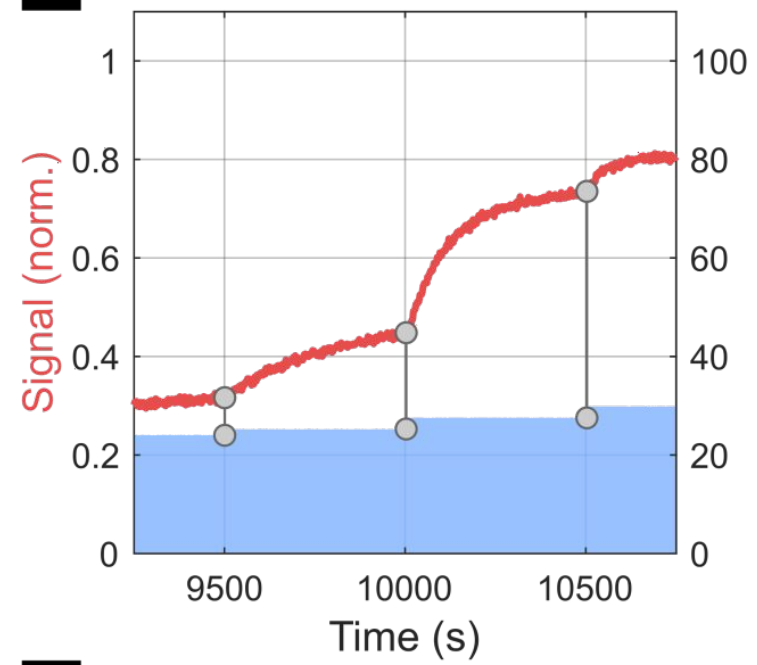

C

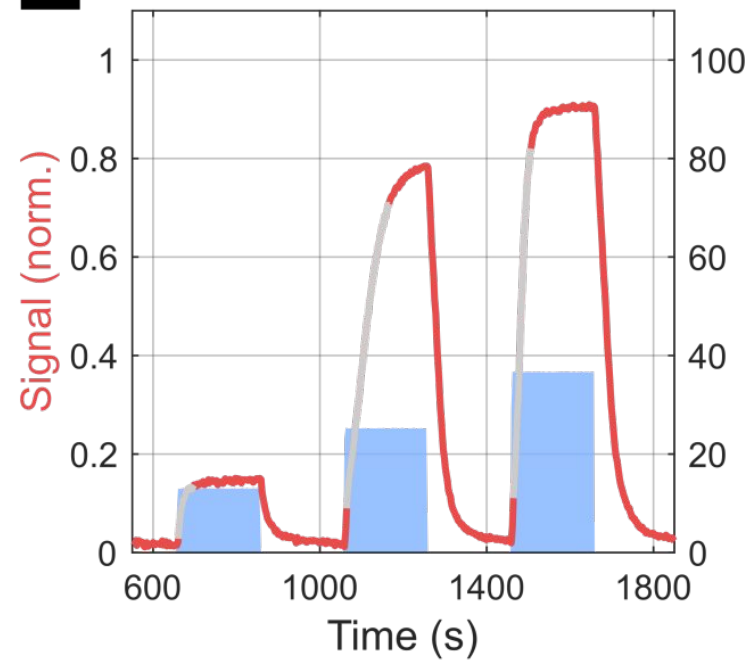

b

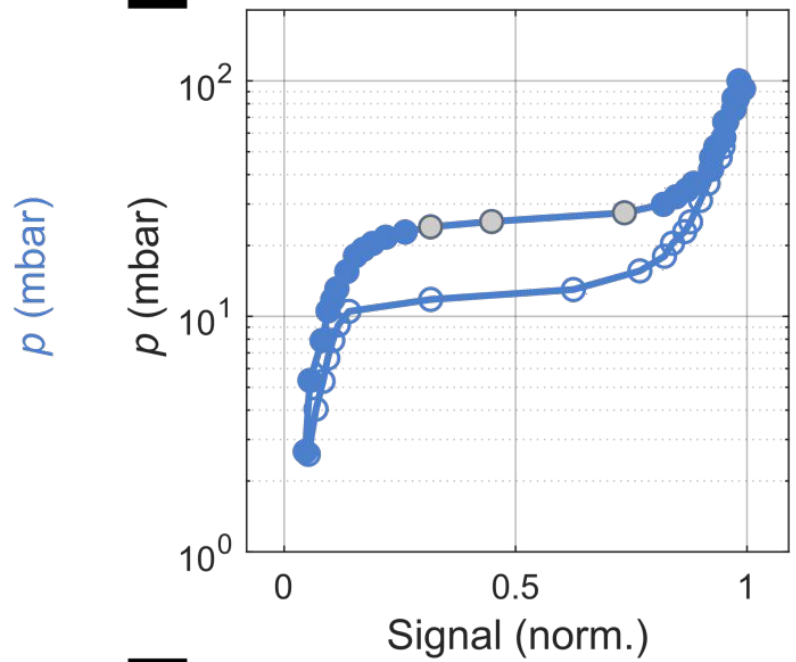

d

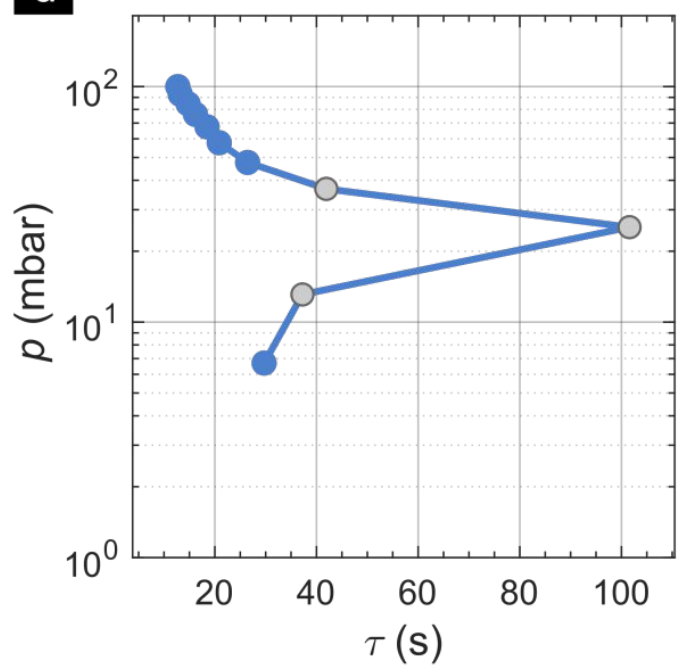

Figure S4. Acquisition of the isothermal data. The data was recorded at $25^{\circ} \mathrm{C}$ with a brightfield microspectroscopy setup and subsequently evaluated at $600 \mathrm{~nm}$. (a) shows parts of the data that is used to determine the pressure-composition isotherm in (b). The interconnected gray circles in (a) correspond to the gray circles in the absorption curve of (b). The gray sections of the signal in (c) indicate the regions that are evaluated to derive the $10-90 \%$ rise times that relate to the gray circles in (d).

The isothermal datasets for the pressure-composition isotherms and the signal response times are acquired with a brightfield microspectroscopy setup (see experimental section). This is necessary since a constant temperature of the sample must be ensured, which requires a temperature control unit that is not integrated in the presented sensor unit. Because of that, the sample is placed in a temperature- 
controlled gas cell that fits into the microspectroscopy setup and thus allows isothermal reflectance measurements. For the acquisition of the pressure-composition isotherms, the hydrogen concentration is monotonically increased and decreased afterward. This is necessary since the equilibrium hydrogen concentration within the palladium nanodisks depends on the previously applied hydrogen partial pressures. The saturation signal is approximated by the signal present shortly before changing to the subsequent hydrogen partial pressure. Although this method tends to slightly underestimate the actual saturation signal, it is still preferred over the estimation by fitting an empirical model to the data. This preference is mainly due to the lack of a simple analytical model that describes the data well in any case. Because of that, model-based estimation of the saturation signal often leads to inaccurate results that must be checked manually and thus is prone to confirmation bias. In future work, the sophisticated model of Teutsch et al. ${ }^{1}$ can be deployed to further improve the evaluation accuracy.

For the determination of the $10-90 \%$ signal rise time, different hydrogen partial pressures are applied starting from a pure nitrogen gas flow. This is done since most sensing applications require the detection of hydrogen in a previously hydrogen-free environment. The $10-90 \%$ signal rise time is selected as figure as merit as it provides the time until the signal has almost saturated and thus the hydrogen concentration can be determined with greatest confidence. Furthermore, other response time metrics that depend on the synchronization of the applied gas pressure and the induced signal change are not applicable since there is an inevitable time delay of the gas pressure at the sample site. This time delay originates from the traveling distance and switching times in the gas mixing system and cannot be determined easily with sufficient accuracy. As is the case for the pressure-composition isotherms, the applied methodology for the response time determination can underestimate the ground-truth, if the signal has not fully saturated. However, for the same reasons as before, no model-based estimation of the saturation signal is applied. 
a
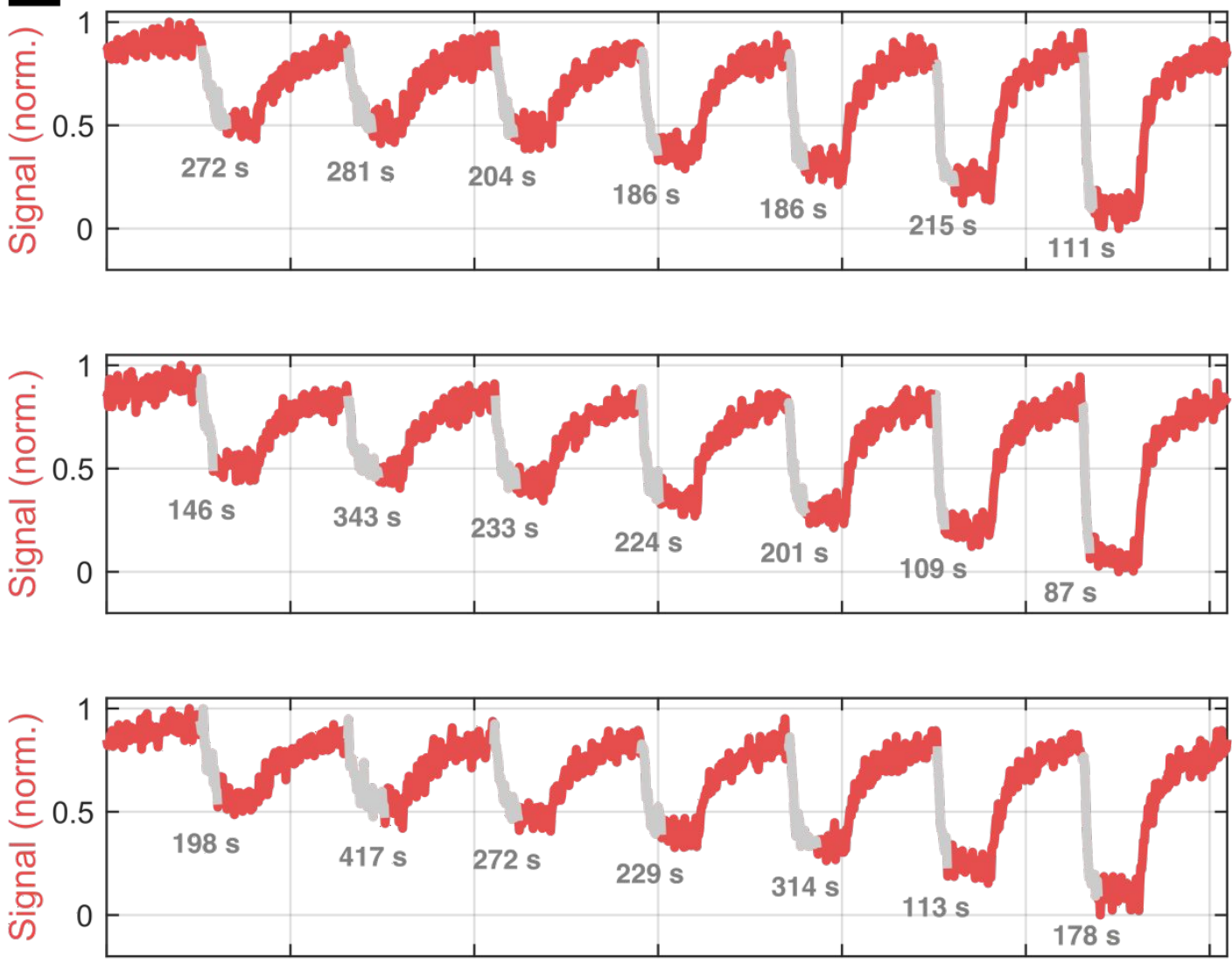

b

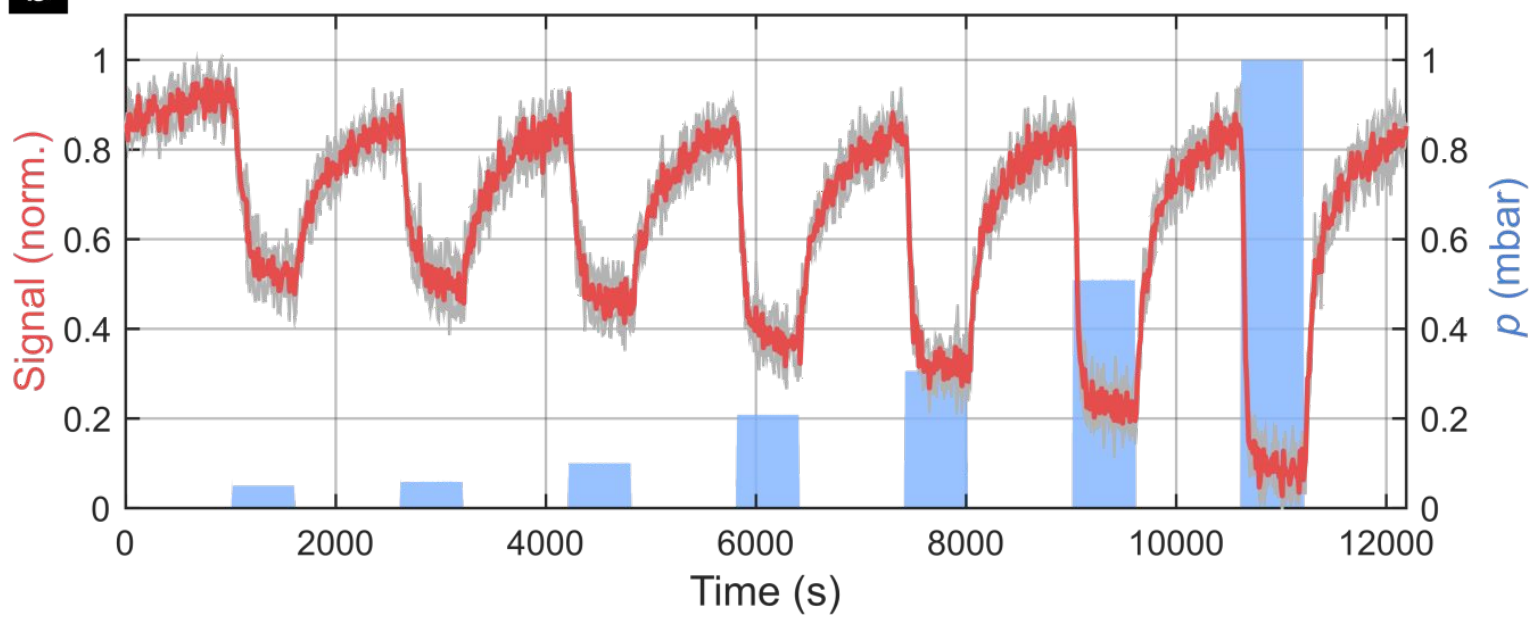

Figure S5. Sensor performance at the lower sensing limit. The data was recorded at room temperature with the compact sensor unit at $780 \mathrm{~nm}$. (a) Three consecutive response curves for the same hydrogen exposure cycle shown in (b) The gray sections of the signal indicate the $10 \%-90 \%$ response time intervals. The mean (red) and standard deviation (gray) of the three response curves is shown in (b). A linear baseline correction and normalization is applied to all curves. Furthermore, the data is downsampled by averaging in blocks of 20 points. The reflectivity decreases upon hydrogen 
exposure since slightly different sample parameters are used here compared to the other measurements presented in this study (PS sphere diameter is 190nm instead of $220 \mathrm{~nm}$ ). This effect is known to occur for specific combinations of the read-out wavelength and resonance lineshape of the reactive sample ${ }^{2}$. 


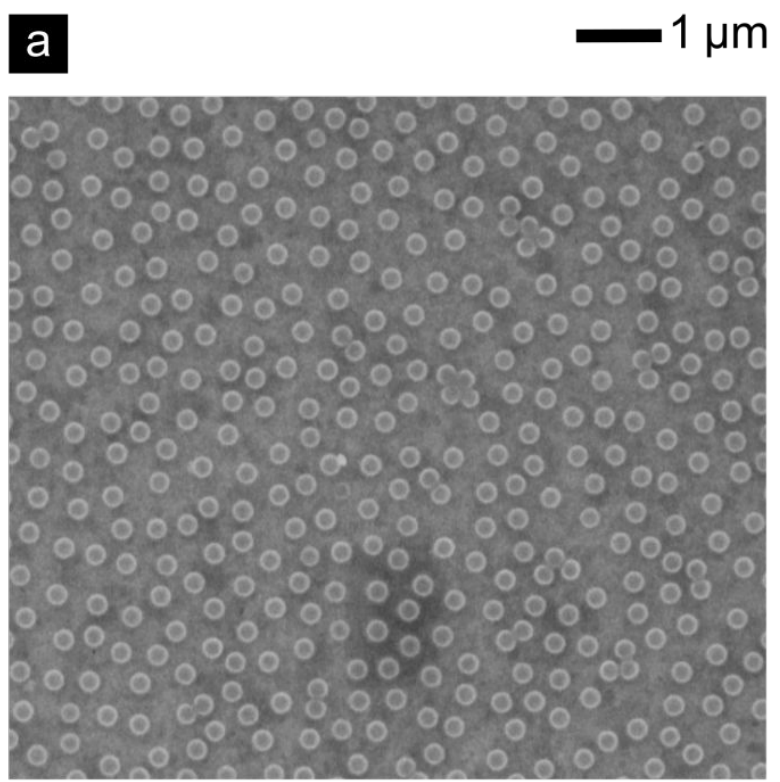

C

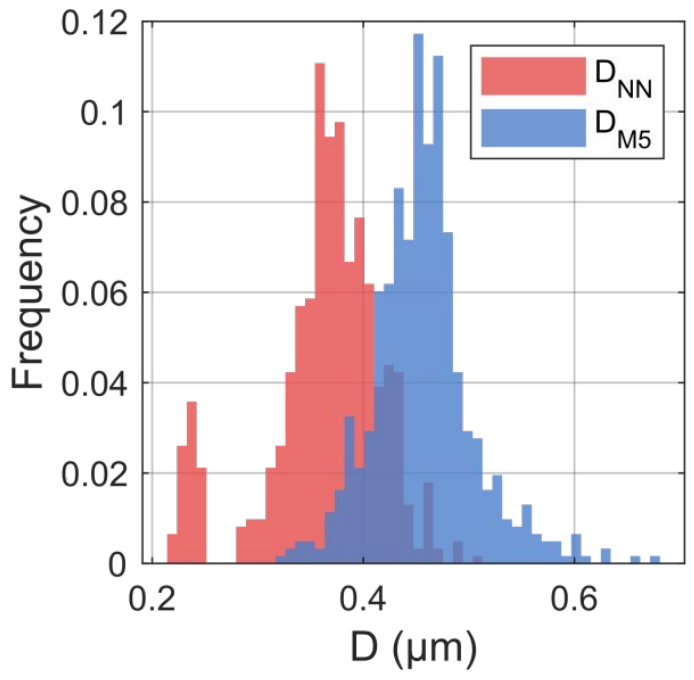

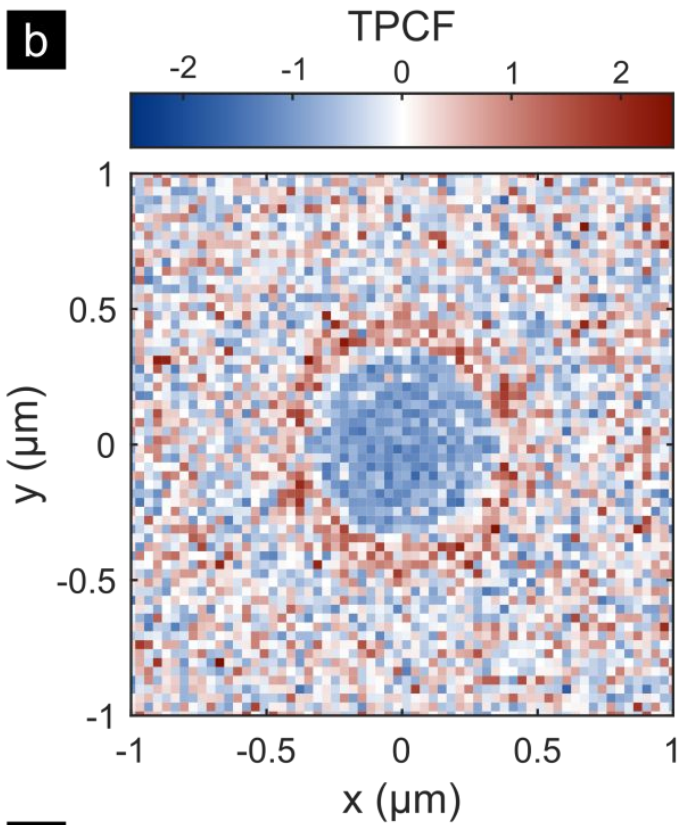

d

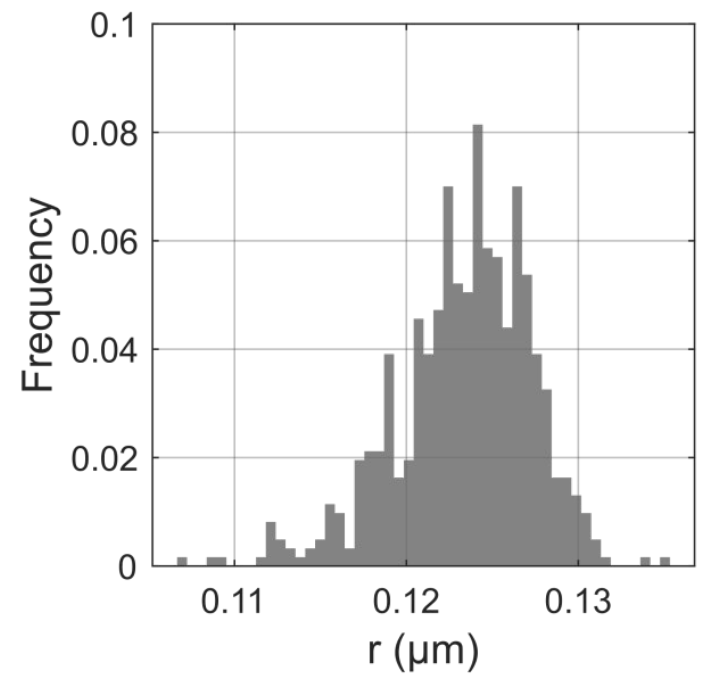

Figure S6. SEM image and structure parameters. (a) Scanning electron microscope (SEM) image of the reactive sample that is fabricated with colloidal etching lithography. (b) The two-point correlation function (TPCF) reveals a highly isotropic distribution with a minimum center-to-center distance of approximately $D=400 \mathrm{~nm}$. (c) This finding is reinforced by the histogram of the nearest neighbor center-to-center distance $\left(D_{N N}\right)$, as well as the mean center-to-center distance to the five nearest neighbors $\left(D_{M 5}\right)$. Particle clustering can be identified at $D_{\mathrm{NN}} \approx 235 \mathrm{~nm}$. (d) The radius distribution allows to determine the mean radius $\mu=123 \mathrm{~nm}$ with a standard deviation of $\sigma=4 \mathrm{~nm}$, assuming a gaussian distribution. This sample was used for the measurements in Fig. 4, Fig. 5, Fig. S1, and Fig. S2. 
Fig. S6 summarizes the most relevant structural details of a typical chemically active sample that is used for the hydrogen sensor. The scanning electron microscope (SEM) image reveals the distribution of the palladium disks on top of the magnesium fluoride spacer layer. While the structure distribution looks random at first sight, the great majority of nanodisks are separated by an isotropic minimum distance. This minimum distance arises from repulsive forces of polystyrene spheres that are utilized during the manufacturing process ${ }^{3}$. The two-point correlation function reinforces this impression and indicates that there is a high probability that two nanodisks are within distance $D$ $\approx 400 \mathrm{~nm}$ and a very low probability that two disks are closer to each other. The twopoint correlation function defines the correlation between the locations of two points (structures) in the sample. Here, the so-called Landy-Szalay estimator ${ }^{4}$ is used for the computation in which positive/negative values indicate a positive/negative correlation. The correlation can be understood in terms of spatial bunching (positive) or antibunching (negative) of the nanostructures and is a vital quantity to understand the scattering properties of nanostructure ensembles ${ }^{5}$. The histogram of the center-tocenter distances confirms the minimum distance of $400 \mathrm{~nm}$ and gives insights on the clustering of nanodisks present in the sample. The mean radius of the nanodisks is determined to be $123 \mathrm{~nm}$ with a standard deviation of about $4 \mathrm{~nm}$. These nanostructure dimensions have empirically shown to be a good compromise between fast diffusion time and optimal optical scattering properties. All structural parameters are derived from the SEM image with the Atherton and Kerbyson phase coding method for circle detection ${ }^{6}$. The exemplary sample used for this study is the same as for the measurements in Fig. 4, 5, S1, and S2. The remaining samples are fabricated in the same batch and have very similar structure parameters as the exemplary sample discussed here. 


\section{References}

(1) Teutsch, T.; Strohfeldt, N.; Sterl, F.; Warsewa, A.; Herkert, E.; Paone, D.; Giessen, H.; Tarin, C. Mathematical Modeling of a Plasmonic Palladium-Based Hydrogen Sensor. IEEE Sens. J. 2018, 18 (5), 1946-1959. https://doi.org/10.1109/JSEN.2017.2786939.

(2) Bagheri, S.; Strohfeldt, N.; Sterl, F.; Berrier, A.; Tittl, A.; Giessen, H. LargeArea Low-Cost Plasmonic Perfect Absorber Chemical Sensor Fabricated by Laser Interference Lithography. ACS Sensors 2016, 1 (9), 1148-1154. https://doi.org/10.1021/acssensors.6b00444.

(3) Walter, R.; Tittl, A.; Berrier, A.; Sterl, F.; Weiss, T.; Giessen, H. Large-Area Low-Cost Tunable Plasmonic Perfect Absorber in the Near Infrared by Colloidal Etching Lithography. Adv. Opt. Mater. 2014, n/a-n/a. https://doi.org/10.1002/adom.201400545.

(4) Landy, S. D.; Szalay, A. S. Bias and Variance of Angular Correlation Functions. Astrophys. J. 1993. https://doi.org/10.1086/172900.

(5) Piechulla, P. M.; Muehlenbein, L.; Wehrspohn, R. B.; Nanz, S.; Abass, A.; Rockstuhl, C.; Sprafke, A. Fabrication of Nearly-Hyperuniform Substrates by Tailored Disorder for Photonic Applications. Adv. Opt. Mater. 2018, 6 (7), 1-10. https://doi.org/10.1002/adom.201701272.

(6) Atherton, T. J.; Kerbyson, D. J. Size Invariant Circle Detection. Image Vis. Comput. 1999, 17 (11), 795-803. https://doi.org/10.1016/s02628856(98)00160-7. 